\title{
Effects of Aster Squamatus (Spreng.) Hieron Ethanolic Extract on Oxidative Stability of Sunflower Oil and Corn Oil
}

\author{
Sebnem Selen Isbilir \\ Trakya University, Turkey \\ Trakya University, Faculty of Science, Department of Chemistry, Edirne, TURKEY \\ sebnemselenisbilir@tralya.edu.tr
}

\section{Extended Abstract}

Lipid peroxidation is one of general problem in foods including edible oils, products containing oil as well as living systems. The measurement of lipid peroxidation products is used to evaluate oxidative stress in vitro or in vivo. Many assays are available to assessment of lipid peroxidation products. The concentrations of primary (conjugated dienes of fatty acids) and secondary (aldehydic compounds, malondialdehyde) products can be measured quantitatively, and the obtained results show the oxidative status of the oil [1]. In recent years, there is an increase in research interested in natural compounds which prevent lipid peroxidation process and act a role as antioxidant.

Aster squamatus (Asteraceae) is an annual wild plant and native to North and South America where the species is invasive to other places. In present study, the ethanol extract of Aster squamatus (Spreng.) Hieron was added to raw sunflower oil and raw corn oil and its effect on oxidative stability of the oils was measured by different assays. The samples of raw oils was also studied with alpha-tocopherol as control. Iodometric titration method and ferric thiocyanate method were used to determine peroxide values (PV) [2]. Anisidine value (AV) test [3] and thiobarbituric acid assay (TBARS) [4] were used to measure secondary oxidation products. Progression of lipid peroxidation was followed for forty two days at intervals by incubating at room temperature. The oil samples containing extract reached a maximum PV after 38 days of storage in the ferric thiocyanate method. And PV of alpha-tocopherol was lower than that of the oil samples with extract. Results of AV measurements of samples were comparable to the result of alpha-tocopherol. TBARS values of the oil samples containing extract increased during the storage period and reached a maximum of $2,2 \pm 0.003 \mu \mathrm{g} / \mathrm{g}$ oil. Their inhibition of TBARS formation were very close to that of alpha-tocopherol. The obtained results from the study recommended that the extract of Aster squamatus (Spreng.) moderately increased oxidative stability of the oils compared with alpha-tocopherol as control compound.

\section{References}

[1] T. N. Semb, "Analytical Methods for Determination of the Oxidative Status in Oils," Ph.D. dissertation, Dept. Biotech., Norwegian Univ., Trondheim, Norway.

[2] S. Ueda, T. Hayashi, and M. Namiki, "Effect of ascorbic acid on lipid autoxidation in a model food system," Agric. Biol. Chem., vol. 50, no. 1, pp. 1-7, 1986.

[3] AOCS Official Method Cd 18-90.

[4] P. J. Ke and A. D. Woyewoda, "Microdetermination of thiobarbituric acid values in marine lipids by a direct spectrophotometric method with a monophasic reaction system," Anal. Chim. Acta, vol. 106, no. 2, pp. 279-284, 1979. 